\title{
Non-Commutative First-Order Sequent Calculus
}

\author{
Makoto Tatsuta \\ National Institute of Informatics \\ 2-1-2 Hitotsubashi, Tokyo 101-8430, Japan \\ tatsuta@nii.ac.jp
}

\begin{abstract}
This paper investigates a non-commutative first-order sequent calculus NCLK. For that, this paper extends a non-commutative positive fragment to a full first-order sequent calculus $\mathrm{LK}^{-}$having antecedent-grouping and no right exchange rule. This paper shows (1) NCLK is equivalent to LJ, (2) NCLK with the exchange rule is equivalent to $\mathrm{LK},(3) \mathrm{LK}^{-}$is equivalent to LJ, and (4) translations between $\mathrm{LK}^{-}$and NCLK.
\end{abstract}

\section{Introduction}

Substructural logics, which are logical systems without some of the contraction rule, the weakening rule, and the exchange rule, have been actively studied in both mathematical logic and computer science. For example, linear logic, which is a logical system without the contraction rule, is successful [3].

We will present a first-order sequent calculus NCLK without the exchange rule, called Non-Commutative First-Order Sequent Calculus. The system has the same language of the first-order classical sequent calculus LK, but has only a restricted set of inference rules. We will show the system is equivalent to the first-order intuitionistic sequent calculus LJ. We will also show the system NCLK becomes equivalent to LK when the exchange rule is added to the system. This shows the exchange rule gives a classical principle. We respect order of formulas in a sequent in the system, but conjunction and disjunction are proved to be commutative according to its inference rules.

Substructural logic without the exchange rule that has non-commutative conjunction and disjunction has been studied, but substructural logic without the exchange rule that has commutative conjunction and disjunction has not been fully studied yet. Recently several interesting results have been discovered for this kind of substructural logic. [1] showed a positive fragment of infinitary Peano Arithmetic without the exchange rule has 1-backtracking game theoretic semantics. [2] showed a positive fragment of infinitary Peano Arithmetic without the exchange rule is equivalent to a positive fragment of infinitary Heyting Arithmetic with the law of excluded middle for $\Sigma_{1}^{0}$-formulas.

This paper will first investigate an underlying logic for those papers. Those papers discussed arithmetic, but we restrict our attention to only its underlying logic, and show the logic itself has a surprising property, that is, the equivalence to LJ. Those papers discussed only a positive system that does not have implication, but we extend a positive fragment to a full logic $\mathrm{LK}^{-}$with implication. The 
system $\mathrm{LK}^{-}$has a sequent having antecedent-grouping and does not have the right exchange rule. Formulas in the antecedent are grouped and structural rules can be used only inside a group. This system is proved to be equivalent to LJ. A key of the equivalence proof is analyzing the minimum length of succedents of sequents in a given proof.

Secondly, we will give the system NCLK, which is obtained from $\mathrm{LK}^{-}$by coding grouping information by the length of a sequence of formulas. We will give translations between NCLK and $\mathrm{LK}^{-}$and show they preserve provability. Combining the equivalence between $\mathrm{LK}^{-}$and LJ, these translations will prove the equivalence between NCLK and LJ. On the other hand, when we add the exchange rule to NCLK, the coding information will be lost and it is proved to become equivalent to LK.

Technical novelties of this paper are (1) the extension of the non-commutative positive fragment $[1,2]$ to the full non-commutative logic $\mathrm{LK}^{-}$with implication, (2) the equivalence between $\mathrm{LK}^{-}$and LJ, (3) the definition of NCLK by coding grouping information by the length of a sequence of formulas, and (4) translations between $\mathrm{LK}^{-}$and NCLK.

[2] showed the fragment of arithmetic without the exchange rule is equivalent to the fragment of intuitionistic arithmetic with the law $\mathrm{EM}_{1}$ of excluded middle for $\Sigma_{1}^{0}$ formulas. On the other hand, our system $\mathrm{LK}^{-}$is equivalent to LJ. We can explain reasons for the difference for $\mathrm{EM}_{1}$ in the following way. The first reason is that the minimum length of the succedents in the sequents in the proof is a key for proving the equivalence. When a proof is given in $\mathrm{LK}^{-}$, we can immediately find the minimum length. On the other hand, when a proof is given in the system in $[1,2]$, since it is an infinitary system, we cannot find the minimum, and instead we can only have flag formulas that are some $\Pi_{1}^{0}$-formulas. For case analysis by flag formulas, [2] needed $\mathrm{EM}_{1}$. The second reason is that we can directly show $\mathrm{LK}^{-}$does not derive $\mathrm{EM}_{1}$, and on the other hand we can drive $\mathrm{EM}_{1}$ in the system in $[1,2]$ by using infinitary logic and true atomic formulas.

$[4,5]$ investigated the sequent calculus obtained from LK by restricting the implication right rule to only intuitionistic sequents and showed the system is equivalent to LJ. Our system NCLK will give another way of restriction to LK so that the resulting system becomes equivalent to LJ.

A potential application of these systems $\mathrm{LK}^{-}$and NCLK will be program extraction, since it is equivalent to first-order intuitionistic logic.

Section 2 defines and discusses $\mathrm{LK}^{-}$. We give definitions of LK and LJ in Section 3. Section 4 proves the implication from $\mathrm{LK}^{-}$to LJ and Section 5 proves the other implication from LJ to $\mathrm{LK}^{-}$. We define and discuss NCLK in Section 6. Section 7 gives the translations between NCLK and $\mathrm{LK}^{-}$, and shows the equivalence between NCLK and LJ.

\section{The System $\mathrm{LK}^{-}$}

Definition 2.1 (language) The language is a first-order language generated from the following symbols. We have variables $x, y, z, \ldots$ We have constants and function symbols. Terms are constructed from variables, constants, and function 
symbols, and denoted by $s, t, \ldots$. We have predicate symbols including 0 -ary predicate symbols $T$ and $\perp$, which mean the truth and the falsity respectively. Atomic formulas are constructed from predicate symbols and terms, and denoted by $a, b, \ldots$. Formulas are defined by

$$
A, B, C, D, \ldots::=a|A \wedge B| A \vee B|A \rightarrow B| \forall x A \mid \exists x A .
$$

We will write $\neg A$ for $A \rightarrow \perp$. $A[t / x]$ is the formula obtained from $A$ by replacing $x$ by $t$.

A sequent is of the form $\Gamma \vdash A_{1}, \ldots, A_{n}$ where $\Gamma$ is a sequence of formulas and $n$ occurrences of the symbol -.

In the sequent $\Gamma_{0},-, \Gamma_{1},-, \Gamma_{2}, \ldots,-, \Gamma_{n} \vdash A_{1}, \ldots, A_{n}$ where $\Gamma_{i}$ is a sequence of formulas and does not contain the symbol -, the group $\Gamma_{0}$ means the initial group, and the $i$-th group $\Gamma_{i}$ corresponds to $A_{i}$.

$\Gamma, \Delta, \Pi, \Sigma, \ldots$ denote a sequence of both formulas and symbols - . We will write $-{ }^{n}$ for $-, \ldots,-(n$ times $) .|\Gamma|$ denotes the number of the formulas in $\Gamma$. $\sharp-\Gamma$ denotes the number of the - symbols in $\Gamma$.

We respect order of formulas in a sequence and a sequent.

We have the following inference rules:

$$
\begin{aligned}
& \frac{\Gamma_{1}, A, \Gamma_{2} \vdash \Delta, A}{}(A x) \quad \frac{\Gamma \vdash \Delta, \top}{\Gamma}(A x \top) \quad \overline{\Gamma_{1}, \perp, \Gamma_{2} \vdash \Delta}(A x \perp) \\
& \frac{\Gamma,-\vdash \Delta, A \wedge B, A \quad \Gamma,-\vdash \Delta, A \wedge B, B}{\Gamma \vdash \Delta, A \wedge B}(\wedge R) \\
& \frac{\Gamma_{1}, A \wedge B, \Gamma_{2}, A \vdash \Delta}{\Gamma_{1}, A \wedge B, \Gamma_{2} \vdash \Delta}(\wedge L 1) \quad \frac{\Gamma_{1}, A \wedge B, \Gamma_{2}, B \vdash \Delta}{\Gamma_{1}, A \wedge B, \Gamma_{2} \vdash \Delta}(\wedge L 2) \\
& \frac{\Gamma,-\vdash \Delta, A \vee B, A}{\Gamma \vdash \Delta, A \vee B}(\vee R 1) \quad \frac{\Gamma,-\vdash \Delta, A \vee B, B}{\Gamma \vdash \Delta, A \vee B}(\vee R 2) \\
& \frac{\Gamma_{1}, A \vee B, \Gamma_{2}, A \vdash \Delta \quad \Gamma_{1}, A \vee B, \Gamma_{2}, B \vdash \Delta}{\Gamma_{1}, A \vee B, \Gamma_{2} \vdash \Delta}(\vee L) \\
& \frac{\Gamma, A \vdash \Delta, A \rightarrow B}{\Gamma \vdash \Delta, A \rightarrow B}(\rightarrow R 1) \quad \frac{\Gamma,-\vdash \Delta, A \rightarrow B, B}{\Gamma \vdash \Delta, A \rightarrow B}(\rightarrow R 2) \\
& \frac{\Gamma_{1}, A \rightarrow B, \Gamma_{2},-\vdash \Delta, A \quad \Gamma_{1}, A \rightarrow B, \Gamma_{2}, B \vdash \Delta}{\Gamma_{1}, A \rightarrow B, \Gamma_{2} \vdash \Delta}(\rightarrow L) \\
& \frac{\Gamma,-\vdash \Delta, \forall x A, A}{\Gamma \vdash \Delta, \forall x A}(\forall R) \quad \frac{\Gamma_{1}, \forall x A, \Gamma_{2}, A[t / x] \vdash \Delta}{\Gamma_{1}, \forall x A, \Gamma_{2} \vdash \Delta}(\forall L) \\
& \frac{\Gamma,-\vdash \Delta, \exists x A, A[t / x]}{\Gamma \vdash \Delta, \exists x A}(\exists R) \quad \frac{\Gamma_{1}, \exists x A, \Gamma_{2}, A \vdash \Delta}{\Gamma_{1}, \exists x A, \Gamma_{2} \vdash \Delta}(\exists L) \\
& \frac{\Gamma \vdash \Delta}{\Gamma,-\vdash \Delta, A}\left(\text { weak R) } \quad \frac{\Gamma \vdash \Delta}{\Gamma, A \vdash \Delta}(\text { weak } L)\right.
\end{aligned}
$$

where the conclusion does not contain free occurrences of $x$ in the rules $(\forall R)$ and $(\exists L)$. 
Intuitive meaning of provable sequents is given as follows: If $\Gamma_{0},-, \Gamma_{1}, \ldots,-, \Gamma_{n} \vdash A_{1}, \ldots, A_{n}$ is provable, then (1) $\Gamma_{0} \vdash$ is true, or (2) $\Gamma_{0},-, \Gamma_{1}, \ldots,-, \Gamma_{i} \vdash A_{i}$ is true for some $i$. Each inference rule is sound by this interpretation. Theorem 4.1 will provide more information.

We explain this system with some examples.

Example 2.2 The first example shows its conjunction is commutative.

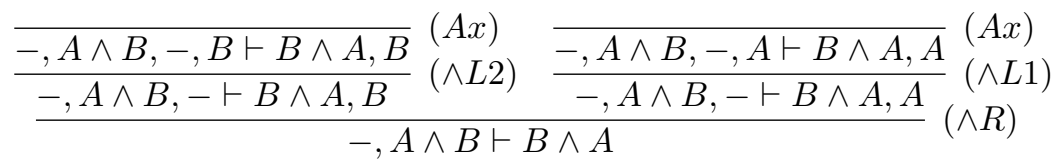

Example 2.3 The next example shows how this system respects the order of formulas. We have three provable sequents

$$
\begin{aligned}
& -, A,-, B \vdash A, \perp, \\
& -, A,-, B \vdash \perp, A, \\
& -, A,-, B \vdash \perp, B .
\end{aligned}
$$

On the other hand the sequent

$$
-, A,-, B \vdash B, \perp
$$

is not provable. The first sequent is provable since the initial and the first groups give the assumption $A$, which proves the first formula $A$. The second sequent is provable since the initial, the first, and the second groups give the assumptions $A, B$, which prove the second formula $A$. The third sequent is provable similarly to the second sequent, since the initial, the first, and the second groups give the assumptions $A, B$, which prove the second formula $B$. Formally the first sequent is proved by

$$
\begin{aligned}
& \frac{\overline{-, A \vdash A}(A x)}{\frac{-, A,-\vdash A, \perp}{-, A,-, B \vdash A, \perp}}(\text { weak } R) \\
& \frac{\text { weak } L)}{}
\end{aligned}
$$

and the second and the third sequents are proved by $(A x)$.

On the other hand, ths fourth sequent is not provable, since we have neither of the following cases: (1) the initial group is empty, which proves the contradiction, nor (2) the initial and the first groups give the assumption $A$, which proves the first formula $B$, nor (3) the initial, the first, and the second groups give the assumptions $A, B$, which prove the second formula $\perp$.

Example 2.4 The third example gives an example with implication.

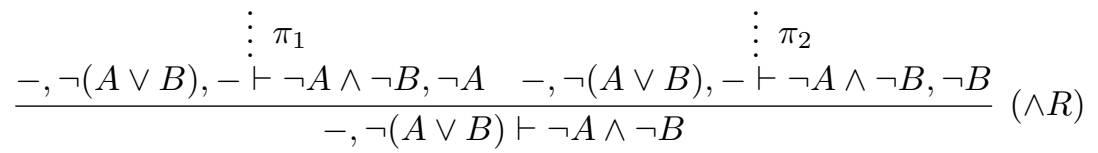


where the proof $\pi_{1}$ is

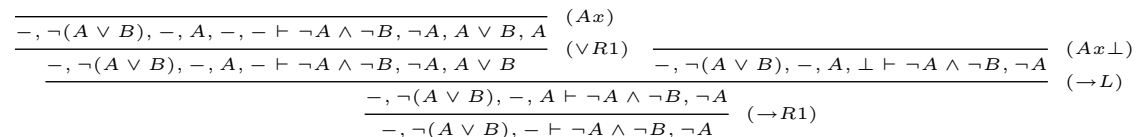

and the proof $\pi_{2}$ is similar to $\pi_{1}$.

We will show some structural rules are admissible in this system. $(\Gamma)_{0}$ is defined to be $\Gamma$ if $\Gamma$ does not contain - $(\Gamma,-, \Pi)_{0}$ is defined to be $\Gamma$ if $\Gamma$ does not contain -.

Proposition 2.5 The following are admissible.

$$
\begin{aligned}
& \frac{\Gamma_{1}, \Gamma_{2} \vdash \Delta}{\Gamma_{1}, A, \Gamma_{2} \vdash \Delta} \text { (weak L2) } \\
& \frac{\Gamma_{1}, \Gamma_{2} \vdash \Delta_{1}, \Delta_{2}}{\Gamma_{1},-, \Gamma_{2} \vdash \Delta_{1}, A, \Delta_{2}}(\text { weak } R 2) \quad\left(\sharp-\Gamma_{2}=\left|\Delta_{2}\right|,\left(\Gamma_{2}\right)_{0}=\phi\right) \\
& \frac{\Gamma \vdash \Delta}{\Pi, \Gamma \vdash \Sigma, \Delta}(\text { weak } R 3) \quad(\sharp-\Pi=|\Sigma|) \quad \frac{\Gamma_{1},-, A, \Gamma_{2} \vdash \Delta}{\Gamma_{1}, A,-, \Gamma_{2} \vdash \Delta}(\text { move }) \\
& \frac{\Gamma_{1}, A, \Gamma_{2}, A, \Gamma_{3} \vdash \Delta}{\Gamma_{1}, A, \Gamma_{2}, \Gamma_{3} \vdash \Delta}(\text { cont } L) \quad \frac{\Gamma_{1}, A, B, \Gamma_{2} \vdash \Delta}{\Gamma_{1}, B, A, \Gamma_{2} \vdash \Delta}(\text { exch } L)
\end{aligned}
$$

These are proved by induction on proofs. For example, in order to prove (weak L2) is admissible, we assume a proof $\pi$ of $\Gamma_{1}, \Gamma_{2} \vdash \Delta$ and construct a proof of $\Gamma_{1}, A, \Gamma_{2} \vdash \Delta$. The idea is just adding $A$ to the antecedent in each sequent in $\pi$. Formally we consider cases according to the last rule used in $\pi$.

Let the rule be $(A x)$ and its conclusion be $\Gamma_{1}^{\prime}, B, \Gamma_{2}^{\prime} \vdash \Delta^{\prime}, B$. We have to show $\Gamma_{1}^{\prime \prime}, B, \Gamma_{2}^{\prime \prime} \vdash \Delta^{\prime}, B$ where $\Gamma_{1}^{\prime \prime}, \Gamma_{2}^{\prime \prime}$ is obtained from $\Gamma_{1}^{\prime}, \Gamma_{2}^{\prime}$ by adding $A$. $\Gamma_{1}^{\prime \prime}, B, \Gamma_{2}^{\prime \prime} \vdash \Delta^{\prime}, B$ is provable by $(A x)$.

Let the rule be $(\vee R 1)$ that derives $\Gamma_{1}, \Gamma_{2} \vdash \Delta^{\prime}, B \vee C$ from $\Gamma_{1}, \Gamma_{2},-\vdash$ $\Delta^{\prime}, B \vee C, B$. We have to show $\Gamma_{1}, A, \Gamma_{2} \vdash \Delta^{\prime}, B \vee C$. By induction hypothesis we have $\Gamma_{1}, A, \Gamma_{2},-\vdash \Delta^{\prime}, B \vee C, B$. By ( $\left.\vee R 1\right)$, we have $\Gamma_{1}, A, \Gamma_{2} \vdash \Delta^{\prime}, B \vee C$. Other cases are proved similarly.

\section{First-Order Sequent Calculi}

This section gives definitions of LK and LJ in a familiar way.

We define the first-order classical sequent calculus LK. The language is defined to be the same as that of $\mathrm{LK}^{-}$except its sequents are of the form $A_{1}, \ldots, A_{n} \vdash B_{1}, \ldots, B_{m} \quad(n, m \geq 0)$ for formulas $A_{1}, \ldots, A_{n}, B_{1}, \ldots, B_{m}$. $\Gamma, \Delta, \Pi, \Sigma, \ldots$ denote a sequence of formulas. 
We have the following inference rules.

$$
\begin{aligned}
& \overline{A \vdash A}(A x) \quad \overline{\vdash \top}(A x \top) \quad \overline{\perp \vdash}(A x \perp) \\
& \frac{\Gamma \vdash \Delta, A \quad \Gamma \vdash \Delta, B}{\Gamma \vdash \Delta, A \wedge B}(\wedge R) \quad \frac{\Gamma, A \vdash \Delta}{\Gamma, A \wedge B \vdash \Delta}(\wedge L 1) \quad \frac{\Gamma, B \vdash \Delta}{\Gamma, A \wedge B \vdash \Delta}(\wedge L 2) \\
& \frac{\Gamma \vdash \Delta, A}{\Gamma \vdash \Delta, A \vee B}(\vee R 1) \frac{\Gamma \vdash \Delta, B}{\Gamma \vdash \Delta, A \vee B}(\vee R 2) \frac{\Gamma, A \vdash \Delta \quad \Gamma, B \vdash \Delta}{\Gamma, A \vee B \vdash \Delta}(\vee L) \\
& \frac{\Gamma, A \vdash \Delta, B}{\Gamma \vdash \Delta, A \rightarrow B}(\rightarrow R) \quad \frac{\Gamma \vdash \Delta, A \quad \Gamma, B \vdash \Sigma}{\Gamma, A \rightarrow B \vdash \Delta, \Sigma}(\rightarrow L) \\
& \frac{\Gamma \vdash \Delta, A}{\Gamma \vdash \Delta, \forall x A}(\forall R) \quad \frac{\Gamma, A[t / x] \vdash \Delta}{\Gamma, \forall x A \vdash \Delta}(\forall L) \\
& \frac{\Gamma \vdash \Delta, A[t / x]}{\Gamma \vdash \Delta, \exists x A}(\exists R) \quad \frac{\Gamma, A \vdash \Delta}{\Gamma, \exists x A \vdash \Delta}(\exists L) \\
& \frac{\Gamma \vdash \Delta}{\Gamma \vdash \Delta, A}(\text { weak } R) \quad \frac{\Gamma \vdash \Delta}{\Gamma, A \vdash \Delta}(\text { weak } L) \\
& \frac{\Gamma \vdash \Delta, A, A}{\Gamma \vdash \Delta, A}(\operatorname{cont} R) \quad \frac{\Gamma, A, A \vdash \Delta}{\Gamma, A \vdash \Delta}(\operatorname{cont} L) \\
& \frac{\Gamma \vdash \Delta_{1}, B, A, \Delta_{2}}{\Gamma \vdash \Delta_{1}, A, B, \Delta_{2}}(\operatorname{exch} R) \quad \frac{\Gamma_{1}, B, A, \Gamma_{2} \vdash \Delta}{\Gamma_{1}, A, B, \Gamma_{2} \vdash \Delta}(\operatorname{exch} L)
\end{aligned}
$$

where the conclusion does not contain free occurrences of $x$ in the rules $(\forall R)$ and $(\exists L)$.

We define the first-order intuitionistic sequent calculus LJ. The language is the same as that of LK except that its sequents are intuitionistic sequents $A_{1}, \ldots, A_{n} \vdash B$ or $A_{1}, \ldots, A_{n} \vdash$. The inference rules are the same as those of LK except that their sequents are restricted to intuitionistic sequents.

\section{Implication from $\mathrm{LK}^{-}$to $\mathrm{LJ}$}

This section proves the direction from $\mathrm{LK}^{-}$to LJ.

For a given proof $\pi$, we define $\|\pi\|$ as the minimum length of the succedents of the sequents in $\pi$. We define $\left(\Gamma_{0},-, \Gamma_{1},-, \Gamma_{2}, \ldots,-, \Gamma_{n}\right)^{+}$as $\Gamma_{0}, \Gamma_{1}, \Gamma_{2}, \ldots, \Gamma_{n}$ if $\Gamma_{0}, \ldots, \Gamma_{n}$ do not contain any - symbol.

Theorem 4.1 If we have a proof of the sequent $\Gamma_{0},-, \Gamma_{1},-, \Gamma_{2}, \ldots,-, \Gamma_{n} \vdash$ $A_{1}, A_{2}, \ldots, A_{n}$ in $L K^{-}$where $n \geq 0$ and $\Gamma_{0}, \ldots, \Gamma_{n}$ do not contain any - symbol, and $i$ is the minimum length of the succedents of the sequents in the proof, then we have the following:

(1) $i=0$ and $\Gamma_{0} \vdash$ is provable in $L J$, or

(2) $i>0$ and $\Gamma_{0}, \ldots, \Gamma_{i} \vdash A_{i}$ is provable in $L J$. 
The idea is analyzing the uppermost sequent $\Gamma \vdash \Delta, A$ with its succedent of length $i$ in the proof. We sketch the proof. Suppose the sequent $\Gamma \vdash \Delta, A$ is such a sequent. Since left rules and $(\rightarrow R 1)$ do not change the length of some succedent, right logical rules except $(\rightarrow R 1)$ decrease the length of succedents by 1 , and $($ weak $R$ ) increases the length of the succedent by 1 , the inference rule deriving $\Gamma \vdash \Delta, A$ must be axioms or right logical rules except $(\rightarrow R 1)$. Then we can show $\Gamma \vdash A$ is provable in LJ. If it is an axiom, $\Gamma \vdash A$ is provable in LJ by the corresponding axiom. If it is a right logical rule, we use induction hypothesis for its subproofs. For example, if it is

$$
\frac{\overline{A, B,-,-\vdash A \wedge B, A}(A x) \overline{A, B,-,-\vdash A \wedge B, B}}{A, B,-\vdash A \wedge B}(A x)
$$

and $i=1$, then the minimum length of succedents in each subproof is 2 . By induction hypothesis for each subproof, we have $A, B \vdash A$ and $A, B \vdash B$ in LJ, so we have $A, B \vdash A \wedge B$ in LJ by $(\wedge R)$.

Since right logical rules except $(\rightarrow R 1)$ decrease the length of succedents by 1 , possible inference rules under $\Gamma \vdash \Delta, A$ are left rules, $(\rightarrow R 1)$, or (weak $R$ ). Those inference rules preserve provability of the $i$-th formula $A$ from the first $i+1$ groups of its antecedent in LJ. Hence, in the conclusion of the given proof, the sequent of the $i$-th formula $A$ from the first $i+1$ groups of its antecedent is provable in LJ.

Theorem 4.2 If $L K^{-}$proves $\Gamma_{0},-, \Gamma_{1} \vdash A$, then LJ proves $\Gamma_{0}, \Gamma_{1} \vdash A$.

Proof. Let $\pi$ be the proof and $i$ be $\|\pi\|$. Then $i$ is 0 or 1 . By Theorem 4.1 with $n=1$, we have (1) $i=0$ and $\Gamma_{0} \vdash$ is provable in LJ, or (2) $i=1$ and $\Gamma_{0}, \Gamma_{1} \vdash A$ is provable in LJ. If $i=1$, we have the claim. If $i=0$, by weakening to $\Gamma_{0} \vdash$, we have the claim.

\section{Implication from $\mathrm{LJ}$ to $\mathrm{LK}^{-}$}

This section proves the implication from LJ to $\mathrm{LK}^{-}$.

Proposition 5.1 If $\Gamma \vdash \Delta$ is provable in LJ where $|\Delta|=0,1$, then $-^{|\Delta|}, \Gamma \vdash \Delta$ is provable in $\mathrm{LK}^{-}$.

The proof idea is simulating each inference rule of LJ by inference rules of $\mathrm{LK}^{-}$. One difference is that a logical rule in $\mathrm{LK}^{-}$has a redundant principal formula. For example, the right conjunction rule in $\mathrm{LK}^{-}$is

$$
\frac{\Gamma,-\vdash \Delta, A \wedge B, A \quad \Gamma,-\vdash \Delta, A \wedge B, B}{\Gamma \vdash \Delta, A \wedge B}(\wedge R)
$$

and on the other hand the right conjunction rule in LJ is

$$
\frac{\Gamma \vdash A \quad \Gamma \vdash B}{\Gamma \vdash A \wedge B}(\wedge R)
$$


This difference is covered by putting $A \wedge B$ by (weak $R 2$ ) in Proposition 2.5. The other difference is the existence of - , which is handled by moving - by (move) in Proposition 2.5.

Theorem 5.2 (Equivalence between $\mathbf{L K}^{-}$and LJ),$- \Gamma \vdash A$ is provable in $L K^{-}$if and only if $\Gamma \vdash A$ is provable in $L J$.

Proof. The implication from the left-hand side to the right-hand side is proved by Theorem 4.2. The implication from the right-hand side to the left-hand side is proved by Proposition 5.1.

\section{Non-Commutative Sequent Calculus NCLK}

This section discusses NCLK and shows it becomes equivalent to LK when we add the exchange rule to it.

We define Non-Commutative First-Order Sequent Calculus NCLK.

Definition 6.1 (NCLK) Its language is the same as that of LK. Note that its sequents are of the form $A_{1}, \ldots, A_{n} \vdash B_{1}, \ldots, B_{m}(n, m \geq 0)$ where $A_{i}, B_{i}$ are formulas. $\Gamma, \Delta, \Pi, \Sigma \ldots$ denote a sequence of formulas.

The inference rules are given as follows.

$$
\begin{aligned}
& \overline{\Gamma_{1}, A, \Gamma_{2} \vdash \Delta, A}(A x) \quad \overline{\Gamma \vdash \Delta, \top}(A x \top) \quad \overline{\Gamma_{1}, \perp, \Gamma_{2} \vdash \Delta}(A x \perp) \\
& \frac{\Gamma, \top \vdash \Delta, A \wedge B, A \quad \Gamma, \top \vdash \Delta, A \wedge B, B}{\Gamma \vdash \Delta, A \wedge B}(\wedge R) \\
& \frac{\Gamma_{1}, A \wedge B, \Gamma_{2}, A \vdash \Delta, D, D}{\Gamma_{1}, A \wedge B, \Gamma_{2} \vdash \Delta, D}(\wedge L 1) \quad \frac{\Gamma_{1}, A \wedge B, \Gamma_{2}, B \vdash \Delta, D, D}{\Gamma_{1}, A \wedge B, \Gamma_{2} \vdash \Delta, D}(\wedge L 2) \\
& \frac{\Gamma, \top \vdash \Delta, A \vee B, A}{\Gamma \vdash \Delta, A \vee B}(\vee R 1) \quad \frac{\Gamma, \top \vdash \Delta, A \vee B, B}{\Gamma \vdash \Delta, A \vee B}(\vee R 2) \\
& \frac{\Gamma_{1}, A \vee B, \Gamma_{2}, A \vdash \Delta, D, D \quad \Gamma_{1}, A \vee B, \Gamma_{2}, B \vdash \Delta, D, D}{\Gamma_{1}, A \vee B, \Gamma_{2} \vdash \Delta, D}(\vee L) \\
& \frac{\Gamma, A \vdash \Delta, A \rightarrow B, A \rightarrow B}{\Gamma \vdash \Delta, A \rightarrow B}(\rightarrow R 1) \quad \frac{\Gamma, \top \vdash \Delta, A \rightarrow B, B}{\Gamma \vdash \Delta, A \rightarrow B}(\rightarrow R 2) \\
& \frac{\Gamma_{1}, A \rightarrow B, \Gamma_{2}, \top \vdash \Delta, D, A \quad \Gamma_{1}, A \rightarrow B, \Gamma_{2}, B \vdash \Delta, D, D}{\Gamma_{1}, A \rightarrow B, \Gamma_{2} \vdash \Delta, D}(\rightarrow L) \\
& \frac{\Gamma, \top \vdash \Delta, \forall x A, A}{\Gamma \vdash \Delta, \forall x A}(\forall R) \quad \frac{\Gamma_{1}, \forall x A, \Gamma_{2}, A[t / x] \vdash \Delta, D, D}{\Gamma_{1}, \forall x A, \Gamma_{2} \vdash \Delta, D}(\forall L) \\
& \frac{\Gamma, \top \vdash \Delta, \exists x A, A[t / x]}{\Gamma \vdash \Delta, \exists x A}(\exists R) \quad \frac{\Gamma_{1}, \exists x A, \Gamma_{2}, A \vdash \Delta, D, D}{\Gamma_{1}, \exists x A, \Gamma_{2} \vdash \Delta, D}(\exists L) \\
& \frac{\Gamma \vdash \Delta}{\Gamma, A \vdash \Delta, B}(\text { sweak }) \quad \frac{\top, \Gamma \vdash \Delta}{\Gamma \vdash \Delta}(\top E) \quad \frac{\Gamma \vdash \perp, \Delta}{\Gamma \vdash \Delta}(\perp E)
\end{aligned}
$$


where the conclusion does not contain free occurrences of $x$ in the rules $(\forall R)$ and $(\exists L)$.

(sweak) means symmetric weakening.

Intuitive meaning of provable sequents is given as follows: If $\Pi, A_{1}, \ldots, A_{n} \vdash$ $B_{1}, \ldots, B_{n}$ is provable, then (1) $\Pi \vdash$ is true, or (2) $\Pi, A_{1}, \ldots, A_{i} \vdash B_{i}$ is true for some $i$. If $A_{1}, \ldots, A_{n} \vdash C_{1}, \ldots, C_{m}, B_{1}, \ldots, B_{n}$ is provable, then (1) $\vdash C_{i}$ is true for some $i$, or (2) $A_{1}, \ldots, A_{i} \vdash B_{i}$ is true for some $i$.

This system is obtained from $\mathrm{LK}^{-}$by coding grouping information by the length of a sequence of formulas. We explain it by example.

Example 6.2 The sequent

$$
A_{1},-, A_{2}, A_{3},-, A_{4},-, A_{5}, A_{6} \vdash B_{1}, B_{2}, B_{3}
$$

in $\mathrm{LK}^{-}$is coded by the sequent

$$
A_{1}, \top, A_{2}, A_{3}, \top, A_{4}, \top, A_{5}, A_{6} \vdash B_{1}, B_{1}, B_{1}, B_{2}, B_{2}, B_{3}, B_{3}, B_{3}
$$

in NCLK. The atomic formula $T$ is used for separating groups. The group T, $A_{5}, A_{6}$ corresponds to $B_{3}, B_{3}, B_{3}$. The group $\top, A_{4}$ corresponds to $B_{2}, B_{2}$. The group $\mathrm{T}, A_{2}, A_{3}$ corresponds to $B_{1}, B_{1}, B_{1}$. We can decode this information by counting formulas from the right to the left on both sides. This translation is formally defined in Definition 7.5.

We explain this system by the same examples as those in Section 2 .

Example 6.3 The first example shows its conjunction is commutative.

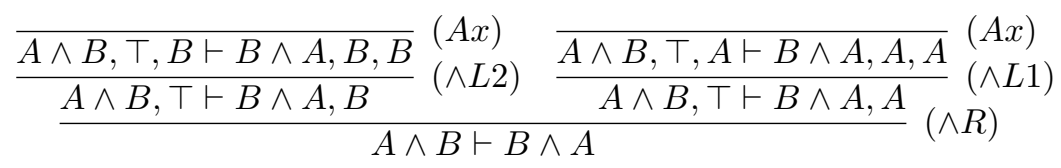

Example 6.4 The next example shows how this system respects the order of formulas. We have three provable sequents

$$
\begin{aligned}
& A, B \vdash A, \perp, \\
& A, B \vdash \perp, A, \\
& A, B \vdash \perp, B .
\end{aligned}
$$

On the other hand the sequent

$$
A, B \vdash B, \perp
$$

is not provable. The first sequent is provable since $A \vdash A$ is true. The second sequent is provable since $A, B \vdash A$ is true. The third sequent is provable since $A, B \vdash B$ is true. Formally the first sequent is proved by

$$
\frac{\overline{A \vdash A}(A x)}{A, B \vdash A, \perp}(\text { sweak })
$$

and the second and the third sequents are proved by $(A x)$. On the other hand, the fourth sequent is not provable, since $A \vdash B$ is not true and $A, B \vdash \perp$ is not true. 
Example 6.5 The third example gives an example with implication.

$$
\frac{\begin{array}{c}
\vdots \\
\pi_{1}
\end{array}}{\neg(A \vee B), \top \vdash \neg A \wedge \neg B, \neg A \quad \neg(A \vee B), \top \vdash \neg A \wedge \neg B, \neg B}(\wedge R)
$$

where the proof $\pi_{1}$ is

$\frac{\frac{\neg(A \vee B), \top, A, \top, \top \vdash \neg A \wedge \neg B, \neg A, \neg A, A \vee B, A}{\neg(A \vee B), \top, A, \top \vdash \neg A \wedge \neg B, \neg A, \neg A, A \vee B} \stackrel{(A x)}{(\vee R 1)} \frac{}{\neg(A \vee B), \top, A, \perp \vdash \neg A \wedge \neg B, \neg A, \neg A, \neg A}}{\frac{\neg(A \vee B), \top, A \vdash \neg A \wedge \neg B, \neg A, \neg A}{\neg(A \vee B), \top \vdash \neg A \wedge \neg B, \neg A}(\rightarrow R 1)}(\rightarrow L)$

and the proof $\pi_{2}$ is similar to $\pi_{1}$.

Remark. (1) Every rule except $(T E)$ and $(\perp E)$ preserves $|\Gamma|-|\Delta|$.

(2) $(\perp E)$ is necessary for making a binary left logical rule for the empty succedent admissible. It is used in the proof of Theorem 7.6. For example, the following is admissible.

$$
\frac{\Gamma_{1}, A \vee B, \Gamma_{2}, A \vdash \quad \Gamma_{1}, A \vee B, \Gamma_{2}, B \vdash}{\Gamma_{1}, A \vee B, \Gamma_{2} \vdash}(\vee L)
$$

(3) ( $T E$ ) is necessary since $\vdash \top \vee \perp, \perp$ would not be provable otherwise, though it is indeed provable by

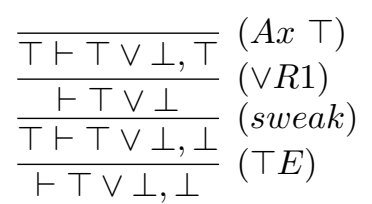

Proposition 6.6 (1) The following are admissible.

$$
\begin{aligned}
& \frac{\Gamma_{1}, \Gamma_{2} \vdash \Delta_{1}, \Delta_{2}}{\Gamma_{1}, A, \Gamma_{2} \vdash \Delta_{1}, B, \Delta_{2}}(\text { sweak }) \quad\left(\left|\Gamma_{2}\right|=\left|\Delta_{2}\right|\right) \\
& \frac{\Gamma_{1}, A, A, \Gamma_{2} \vdash \Delta_{1}, B, B, \Delta_{2}}{\Gamma_{1}, A, \Gamma_{2} \vdash \Delta_{1}, B, \Delta_{2}}(\text { scont }) \quad\left(\left|\Gamma_{2}\right|=\left|\Delta_{2}\right|\right) \\
& \frac{\Gamma_{1}, \Gamma_{2} \vdash \Delta}{\Gamma_{1}, A, \Gamma_{2} \vdash \Delta}(\text { weak } L) \quad \frac{\Gamma \vdash \Delta}{\Gamma \vdash \perp, \Delta}(\perp I) \quad \frac{\Gamma_{1}, \top, \Gamma_{2} \vdash \Delta}{\Gamma_{1}, A, \Gamma_{2} \vdash \Delta}(\text { replace } L)
\end{aligned}
$$

(2) The following is admissible.

$$
\frac{\Gamma \vdash \Delta_{1}, A, A, \Delta_{2}}{\Gamma \vdash \Delta_{1}, A, \Delta_{2}}(\text { cont } R)
$$

(3) The following is admissible.

$$
\frac{\Gamma_{1}, \top, A, \Gamma_{2} \vdash \Delta_{1}, B, B, \Delta_{2}}{\Gamma_{1}, A, \Gamma_{2} \vdash \Delta_{1}, B, \Delta_{2}}(\top E 2) \quad\left(\left|\Gamma_{2}\right|=\left|\Delta_{2}\right|\right)
$$


The claims in (1) are proved by induction on the proof. The claim (2) is proved by induction on the proof by using (weak $L$ ) in (1). The claim (3) is proved by (scont) and (replace $L$ ) in (1).

We define the system NCLK+EX as NCLK with $($ exch $L)$ and $($ exch $R)$.

$$
\frac{\Gamma \vdash \Delta_{1}, A, B, \Delta_{2}}{\Gamma \vdash \Delta_{1}, B, A, \Delta_{2}}(\operatorname{exch} R) \quad \frac{\Gamma_{1}, A, B, \Gamma_{2} \vdash \Delta}{\Gamma_{1}, B, A, \Gamma_{2} \vdash \Delta}(\operatorname{exch} L)
$$

Proposition 6.7 The following are admissible in $N C L K+E X$.

$$
\frac{\Gamma, A, A \vdash \Delta}{\Gamma, A \vdash \Delta}(\text { cont } L) \quad \frac{\Gamma \vdash \Delta}{\Gamma \vdash \Delta, A}(\text { weak } R)
$$

Proof. (cont $L)$ is proved by

$$
\begin{aligned}
& \frac{\Gamma, A, A \vdash \Delta}{\Gamma, A, A \vdash \perp, \perp, \Delta}(\perp I) \text { twice } \\
& \frac{\frac{\Gamma, A, A \vdash \Delta, \perp, \perp}{\Gamma, A \vdash \Delta, \perp}}{\left.\frac{\Gamma}{\Gamma, A c h} R\right) \text { several times }} \\
& \frac{(\text { scont })}{\Gamma, A \vdash \perp, \Delta}(\text { exch } R) \text { several times } \\
& (\perp E)
\end{aligned}
$$

$($ weak $R$ ) is proved by

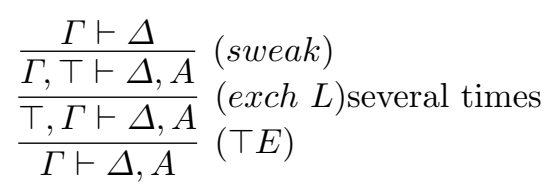

We will write $\Gamma \vdash_{T} \Delta$ to denote that the sequent $\Gamma \vdash \Delta$ is provable in the system $T$.

Theorem 6.8 (Equivalence between NCLK+EX and LK)

$\Gamma \vdash N C L K+E X \Delta$ if and only if $\Gamma \vdash{ }_{L K} \Delta$.

Proof. From the right-hand side to the left-hand side. The claim is proved by induction on the proof.

If the last rule is a logical rule, then add some formulas by weakening in Propositions 6.6 and 6.7 and use the corresponding logical rule. We give some interesting cases.

Case $(\rightarrow R)$. We suppose

$$
\frac{\Gamma, A \vdash \Delta, B}{\Gamma \vdash \Delta, A \rightarrow B}(\rightarrow R)
$$

Then we have

$$
\begin{gathered}
\vdots \\
\frac{I H}{\Gamma, A \vdash \Delta, B} \\
\frac{\frac{\Gamma, A, \top \vdash \Delta, A \rightarrow B, A \rightarrow B, B}{\Gamma, A \vdash \Delta, A \rightarrow B, A \rightarrow B}}{\frac{\Gamma \vdash \Delta, A \rightarrow B}{\Gamma}}(\rightarrow R 1) \\
(\rightarrow R 2)
\end{gathered}
$$


Case $(\rightarrow L)$. We suppose

$$
\frac{\Gamma \vdash \Delta, A \quad \Gamma, B \vdash \Sigma}{\Gamma, A \rightarrow B \vdash \Delta, \Sigma}(\rightarrow L)
$$

Then we have

$$
\begin{aligned}
& \vdots I H \quad \vdots I H \\
& \frac{\frac{\Gamma \vdash \Delta, A}{\Gamma, A \rightarrow B, \top \vdash \Delta, \Sigma, \perp, A}(W) \frac{\Gamma, B \vdash \Sigma}{\Gamma, A \rightarrow B, B \vdash \Delta, \Sigma, \perp, \perp}}{\frac{\Gamma, A \rightarrow B \vdash \Delta, \Sigma, \perp}{\Gamma, A \rightarrow B \vdash \perp, \Delta, \Sigma}(\text { exch } R) \text { several times }}(\rightarrow L)
\end{aligned}
$$

where $(W)$ denotes several steps of (weak $L)$, (weak $R)$, and (exch $R$ ).

If the last rule is a structural rule, then it is covered by Propositions 6.6 and 6.7

From the left-hand side to the right-hand side. It is proved by induction on a proof since every rule is sound in LK.

\section{Translations between $\mathrm{LK}^{-}$and NCLK}

This section shows the equivalence between $\mathrm{LK}^{-}$and NCLK by giving translations which preserve provability.

First we prepare several admissible rules in $\mathrm{LK}^{-}$for the equivalence proof.

Proposition 7.1 The following are admissible in $L K^{-}$.

$$
\begin{aligned}
& \frac{\Gamma_{1}, \top, \Gamma_{2} \vdash \Delta}{\Gamma_{1}, \Gamma_{2} \vdash \Delta}(\top E) \quad \frac{\Gamma_{1},-, \Gamma_{2} \vdash \Delta_{1}, A, A, \Delta_{2}}{\Gamma_{1}, \Gamma_{2} \vdash \Delta_{1}, A, \Delta_{2}}(\text { cont } R) \quad\left(\sharp-\Gamma_{2}=\left|\Delta_{2}\right|\right) \\
& \frac{\Gamma_{1},-, \Gamma_{2} \vdash \Delta_{1}, \perp, \Delta_{2}}{\Gamma_{1}, \Gamma_{2} \vdash \Delta_{1}, \Delta_{2}}(\perp E) \quad\left(\sharp-\Gamma_{2}=\left|\Delta_{2}\right|\right)
\end{aligned}
$$

They are proved by induction on the proof.

We give a translation from NCLK to $\mathrm{LK}^{-}$. To translate $\Gamma \vdash \Delta$, we insert the same number of - symbols as $|\Delta|$ into $\Gamma$ in front of each formula from the rightmost formula of $\Gamma$. For example, the sequent $A_{1}, A_{2}, A_{3}, A_{4} \vdash B_{1}, B_{2}$ in NCLK is translated into the sequent $A_{1}, A_{2},-, A_{3},-, A_{4} \vdash B_{1}, B_{2}$ in $\mathrm{LK}^{-}$.

Definition 7.2 (Translation from NCLK to LK ${ }^{-}$) $\Gamma \quad \vdash \quad \Delta \quad$ is mapped to $\Gamma^{-|\Delta|} \vdash \Delta$, where $\left(\Gamma_{0}, A_{1}, A_{2}, \ldots, A_{n}\right)^{-n}$ is defined as $\Gamma_{0},-, A_{1},-, A_{2}, \ldots,-, A_{n}$ and $\left(A_{1}, A_{2}, \ldots, A_{m}\right)^{-n}(m<n)$ is defined as $-{ }^{n-m},-, A_{1},-, A_{2}, \ldots,-, A_{m}$.

Theorem 7.3 $\Gamma \vdash_{N C L K} \Delta$ implies $\Gamma^{-|\Delta|} \vdash_{L K^{-}} \Delta$. 
Proof. By induction on the proof. Cases are considered according to the last rule.

If the last rule is $(A x),(A x \top)$, and $(A x \perp)$, it is proved by $(A x),(A x \top)$, and $(A x \perp)$ respectively.

If the last rule is a right logical rule except $(\rightarrow R 1)$, we first use $(T E)$ in Proposition 7.1, and then use the corresponding logical rule.

If the last rule is a left logical rule except $(\rightarrow L)$ or $(\rightarrow R 1)$, we first use $($ cont $R$ ) in Proposition 7.1, and then use the corresponding logical rule.

If the last rule is $(\rightarrow L)$, we suppose

$$
\frac{\Gamma_{1}, A \rightarrow B, \Gamma_{2}, \top \vdash \Delta, D, A \quad \Gamma_{1}, A \rightarrow B, \Gamma_{2}, B \vdash \Delta, D, D}{\Gamma_{1}, A \rightarrow B, \Gamma_{2} \vdash \Delta, D}(\rightarrow L)
$$

Let $n$ be $|\Delta, D|$. We have

$$
\begin{array}{cc}
\vdots & \vdots H \\
\frac{\left(\Gamma_{1}, A \rightarrow B, \Gamma_{2}\right)^{-n},-, \top \vdash \Delta, D, A}{\left(\Gamma_{1}, A \rightarrow B, \Gamma_{2}\right)^{-n},-\vdash \Delta, D, A}(\top E) & \frac{\left(\Gamma_{1}, A \rightarrow B, \Gamma_{2}\right)^{-n},-, B \vdash \Delta, D, D}{\left(\Gamma_{1}, A \rightarrow B, \Gamma_{2}\right)^{-n}, B \vdash \Delta, D} \\
\left(\Gamma_{1}, A \rightarrow B, \Gamma_{2}\right)^{-n} \vdash \Delta, D & (\text { cont } R)
\end{array}
$$

If the last rule is (sweak), we have

$$
\frac{\frac{\Gamma \vdash \Delta}{\Gamma,-\vdash \Delta, B}(\text { weak } R)}{\Gamma,-, A \vdash \Delta, B}(\text { weak } L)
$$

If the last rule is $(\top E)$ and $(\perp E)$, the claim is proved by $(\top E)$ and $(\perp E)$ in Proposition 7.1 respectively.

Example 7.4 The NCLK-proof of $A \wedge B \vdash B \wedge A$ in Example 6.3 is translated into the $\mathrm{LK}^{-}$-proof

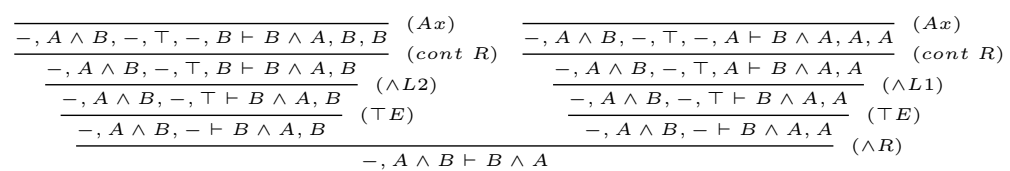

Next, we define a translation from $\mathrm{LK}^{-}$to NCLK. To translate $\Gamma \vdash \Delta$, we replace - by $T$ in $\Gamma$, and the succedent is produced from $\Delta$ by multiplying the $i$-th formula by $n_{i}+1$ when the $i$-th group in $\Gamma$ has $n_{i}$ formulas. An example is given in Example 6.2. $A^{n}$ denotes $A, \ldots, A$ ( $n$ times).

Definition 7.5 (Translation from $\mathbf{L K}^{-}$to NCLK) $\Gamma \vdash \Delta$ is mapped to $\Gamma^{\top} \vdash \Delta^{\Gamma}$, where $\Gamma^{\top}$ is defined as $\Gamma_{0}, \top, \Gamma_{1}, \top, \Gamma_{2}, \ldots, \top, \Gamma_{n}$ and $\left(A_{1}, \ldots, A_{n}\right)^{\Gamma}$ is defined as $A_{1}^{\left|\Gamma_{1}\right|+1}, A_{2}^{\left|\Gamma_{2}\right|+1}, \ldots, A_{n}^{\left|\Gamma_{n}\right|+1}$ if $\Gamma$ is $\Gamma_{0},-, \Gamma_{1},-, \Gamma_{2}, \ldots,-, \Gamma_{n}$ and $\Gamma_{i}$ does not contain -.

Theorem 7.6 $\Gamma \vdash_{L K^{-}} \Delta$ implies $\Gamma^{\top} \vdash_{N C L K} \Delta^{\Gamma}$. 
Proof. By induction on the proof.

Case $(\rightarrow L)$. We suppose

$$
\frac{\Gamma_{1}, A \rightarrow B, \Gamma_{2},-\vdash \Delta, A \quad \Gamma_{1}, A \rightarrow B, \Gamma_{2}, B \vdash \Delta}{\Gamma_{1}, A \rightarrow B, \Gamma_{2} \vdash \Delta}(\rightarrow L)
$$

Case 1. $\Delta$ is not empty. Let $D$ be the last formula of $\Delta$ and $\Gamma$ be $\Gamma_{1}, A \rightarrow B, \Gamma_{2}$. We use $(\rightarrow L)$ with induction hypothesis in the following way.

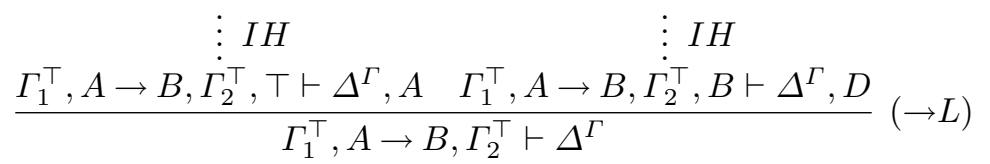

Case 2. $\Delta$ is empty.

$$
\begin{aligned}
& \vdots I H \quad \vdots I H \\
& \frac{\frac{\Gamma_{1}, A \rightarrow B, \Gamma_{2}, \top \vdash A}{\Gamma_{1}, A \rightarrow B, \Gamma_{2}, \top \vdash \perp, A}(\perp I) \quad \frac{\Gamma_{1}, A \rightarrow B, \Gamma_{2}, B \vdash}{\Gamma_{1}, A \rightarrow B, \Gamma_{2}, B \vdash \perp, \perp}}{\frac{\Gamma_{1}, A \rightarrow B, \Gamma_{2} \vdash \perp}{\Gamma_{1}, A \rightarrow B, \Gamma_{2} \vdash}(\perp E)}(\rightarrow L) \text { twice }
\end{aligned}
$$

Case $(\wedge L 1)$ and $\Delta=\phi$.

$$
\begin{aligned}
& \frac{\Gamma_{1}, A \wedge B, \Gamma_{2}, A \vdash}{\Gamma_{1}, A \wedge B, \Gamma_{2}, A \vdash \perp, \perp}(\perp I) \text { twice } \\
& \frac{\Gamma_{1}, A \wedge B, \Gamma_{2} \vdash \perp}{\Gamma_{1}, A \wedge B, \Gamma_{2} \vdash}(\perp E)
\end{aligned}
$$

Cases of other left logical rules with the empty succedent are similarly proved.

Case (weak $R$ ).

$$
\frac{\Gamma^{\top} \vdash \Delta^{\Gamma}}{\Gamma^{\top}, \top \vdash \Delta^{\Gamma}, A}(\text { sweak })
$$

Case (weak $L$ ). If $\Delta$ is not empty, this is proved by (sweak). If $\Delta$ is empty, this is proved by (weak $L)$ in Proposition 6.6.

Example 7.7 The $\mathrm{LK}^{-}$-proof of -, $A \wedge B \vdash B \wedge A$ in Example 2.2 is translated into the NCLK-proof

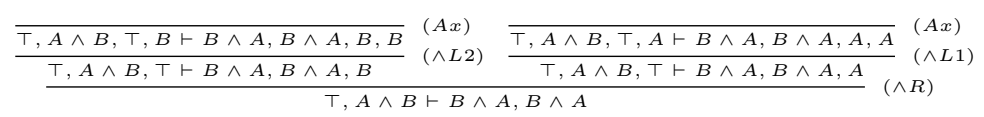

Actually these two translations are the inverses of each other with respect to provability. Let the sequent $\Gamma \vdash \Delta$ in NCLK be translated into the sequent $(\Gamma \vdash \Delta)^{1}$ in $\mathrm{LK}^{-}$and the sequent $\Pi \vdash \Sigma$ in $\mathrm{LK}^{-}$be translated into the sequent $(\Pi \vdash \Sigma)^{2}$ in NCLK. By ( $\left.T E 2\right)$ in Proposition 6.6 (3) and ( $\left.T E\right)$, we can show that if $\left((\Gamma \vdash \Delta)^{1}\right)^{2}$ is provable in NCLK, then $\Gamma \vdash \Delta$ is provable in NCLK. By (cont $R$ ) and $(T E)$ in Proposition 7.1, we can also show that if $\left((\Pi \vdash \Sigma)^{2}\right)^{1}$ is provable in $\mathrm{LK}^{-}$, then $\Pi \vdash \Sigma$ is provable in $\mathrm{LK}^{-}$.

Finally we show the equivalence. 
Theorem 7.8 (Equivalence between NCLK and LJ) $\Gamma \vdash_{N C L K} A$ if and only if $\Gamma \vdash L J A$.

Proof. From the left-hand side to the right-hand side.

By Theorem 7.3, $\Gamma^{-1} \vdash_{\mathrm{LK}^{-}} A$. By Theorem 4.2, $\Gamma \vdash_{\mathrm{LJ}} A$.

From the right-hand side to the left-hand side.

By Proposition 5.1,,$- \Gamma \vdash_{\mathrm{LK}^{-}} A$. By Theorem 7.6, Т, $\Gamma \vdash_{\mathrm{NCLK}} A^{|\Gamma|+1}$. By $(T E)$ and (cont $R$ ) in Proposition $6.6(2)$, we have $\Gamma \vdash_{\text {NCLK }} A$.

\section{Concluding Remarks}

We gave the non-commutative first-order sequent calculus NCLK and showed that it is equivalent to the first-order intuitionistic sequent calculus LJ. We also showed that it becomes equivalent to the first-order classical sequent calculus LK when we add the exchange rule to the system. In order to do that, we extended the non-commutative positive fragment to the system $\mathrm{LK}^{-}$having antecedentgrouping and no right exchange rule. We showed the equivalence between $\mathrm{LK}^{-}$ and LJ. We also gave translations between $\mathrm{LK}^{-}$and NCLK.

The cut elimination theorem holds in $\mathrm{LK}^{-}$. It is proved by extending Theorem 4.1 and using the cut elimination theorem for LJ. The cut elimination theorem is also proved to hold for NCLK by using that for $\mathrm{LK}^{-}$and the translations.

The systems NCLK and $\mathrm{LK}^{-}$give a starting point for research on logical systems based on non-commutative sequents. They will clarify those systems and enable us to extend them.

\section{Acknowledgments}

We would like to thank Prof. Stefano Berardi and Prof. Kazushige Terui for discussions and comments. We would also like to thank the anonymous referees for valuable comments.

\section{References}

1. S. Berardi and Y. Yamagata, A sequent calculus for Limit Computable Mathematics, Annals of Pure and Applied Logic 153 (1-3) (2008) 111-126.

2. S. Berardi and M. Tatsuta, Positive Arithmetic without Exchange is a Subclassical Logic, In: Proceedings of the Fifth Asian Symposium on Programming Languages and Systems (APLAS 2007), Lecture Notes in Computer Science 4807 (2007) 271285.

3. J.Y. Girard, Linear logic, Theoretical Computer Science 50 (1) (1987) 1-102.

4. S. Maehara, Eine Darstellung der intuitionistischen Logik in der Klassischen, Nagoya Mathematical Journal 7 (1954) 45-64.

5. H. Nakano, A Constructive Formalization of the Catch and Throw Mechanism, In: Proceedings of Seventh Annual IEEE Symposium on Logic in Computer Science (1992) 82-89. 